\title{
Green Tea/Licorice Extract-based Antioxidant Solution
}

National Cancer Institute

\section{Source}

National Cancer Institute. Green Tea/Licorice Extract-based Antioxidant Solution. NCI

Thesaurus. Code C158600.

A nutritional supplement containing a variety of antioxidants, vitamins, minerals and amino acids, including glycyrrhizic acid, epigallocatechin gallate (EGCG), zinc, vitamins B5, B6 and B12, vitamin C (ascorbic acid), folic acid, malic acid, glucosamine, arginine, and glycine, with potential immunomodulating, anti-inflammatory, protective, and antineoplastic activities. Upon oral administration, the antioxidants in the solution modulate certain enzymes involved in inflammation and oxidative stress and downregulate certain pro-inflammatory mediators. They also scavenge free radicals. This protects against inflammation- and reactive oxygen species (ROS)-induced cellular damage. In addition, this formulation may also inhibit various signal transduction pathways involved in inflammation and cancer, may suppress the growth of susceptible tumor cells, induce tumor cell cycle arrest, induce apoptosis and reduce angiogenesis and metastasis. 